\title{
CONTRIBUIÇÃO PARA A HISTÓRIA DA NEUROLOGIA EM SÃO PAULO
}

\author{
Paulo Pinto Pupo *
}

A eventualidade de ter sido levado a fazer um discurso regimental na Academia de Medicina de São Paulo nos trouxe a idéia de coletar alguns dados relativos à história da neurologia em São Paulo, história essa que se nos apresenta bastante rica de iniciativas e de realizações. São êsses elementos, enunciados em ordem cronológica, os que constituem o conteúdo dêste trabalho. Dada a extensão da bibliografia neurológica paulista resolvemos documentar êste simplesmente com os trabalhos que nos parecem pioneiros em cada um dos setores aqui abordados. A evolução da neurologia nestes últimos 30 anos, neurologia no sentido lato da palavra, englobando todos os seus ramos afins, nos levou também a fazer uma exortação no sentido da formação de Institutos de Neurologia em nosso meio, com a finalidade de melhor propiciar elementos para a assistência aos doentes neurológicos, assim como ambiente para os estudos de terapêutica e de profilaxia, concentrando esforços no setor material e possibilitando o trabalho de conjunto a essa plêiade de entusiastas cientistas que vêm se formando de modo tão evidente.

Remontamos às primeiras décadas dêste século, quando Arnaldo criava nossa Faculdade de Medicina, para assinalar os grandes clínicos de então, que já de modo tão proficiente exerciam a neurologia clínica. São da reminiscência de nossos mestres os trabalhos de Almeida Prado ${ }^{5,11}$ e de Ovídio Pires de Campos ${ }^{2,3}$, as aulas minuciosas pautadas na neurologia francesa clássica, as discussões que então se feriam nas vetustas enfermarias da Santa Casa de Misericórdia de São Paulo a respeito de diagnósticos clínicos e, já naquele tempo, indicações de neurocirurgia, quando $W$. Seng 1, ? e A. C. Camargo ${ }^{8}$ se aventuravam às primeiras craniotomias em nossa terra.

Coube a Franco da Rocha, ao fundar esta notável organização que foi - Hospital de Juqueri, lançar os alicerces para um ambiente de estudo mais científico das moléstias do cérebro e, com êle, a seu discípulo e sucessor - A. C. Pacheco e Silva - ao organizar, em $1926^{6,9,12,19}$, o laboratório de neuropatologia e do líquido cefalorraqueano naquele Hospital e ao instituir o regime de internato para os estudantes da Faculdade de Medicina, criar o primeiro centro que se constituiu em escola de neuropatologia e de neuropsiquiatria em nosso meio. A sua iniciativa de trazer para aqui o neuropatologista de renome universal - Constantin Chipiakoff - deu ainda maior ênfase à organização e o Hospital de Juqueri se tornou, já naquela época (1924), o centro de atração de inúmeros estudiosos de nossa

* Docente-livre de Neurologia na Fac. Med. Univ. São Paulo; assistente da Clínica Neurológica da Escola Paulista de Medicina (Prof. Paulino W. Longo). 
Faculdade de Medicina. Dessa escola saíram os trabalhos pioneiros. As "Memórias do Hospital de Juqueri" e os "Arquivos da Assistência a Psicopatas" atestam-no de sobêjo.

Entretanto, mérito ímpar cabe a Enjolras Vampré como fundador da neurologia paulista. Foi êste médico sergipano, diplomado na então principal escola médica do País - a Faculdade de Medicina da Bahia - que tão cedo se radicou em São Paulo, o homem que criou dentro da Faculdade de Medicina o ensino da neurologia clínica, que reuniu em tôrno de si discípulos inúmeros e entusiastas, que soube com a magia da sua palavra, da sua mímica, de seu entusiasmo pela neurologia clínica, quem criou o que verdadeiramente se pode denominar a Escola Neurológica Paulista. Dêle descendem, em gerações sucessivas, todos os neurologistas e neurocirurgiōes que aqui trabalham, evoluindo com o progredir da ciência neurológica, trazendo consigo os ensinamentos do Mestre, procurando mais e mais conhecer o porque dos fatos clínicos, a razão de cada um dos sinais e dos sintomas, o raciocínio lógico para o mais completo diagnóstico, os conhecimentos os mais modernos para a mais eficaz terapêutica e, sobretudo, guardando sempre dentro de si a consciência da existência de um doente a ser compreendido, de uma pessoa a ser aliviada de seus sofrimentos, de uma familia a ser consolada nos momentos mais cruciantes quando a moléstia, por vêzes incurável, vai avançando com o seu efeito destruidor. Estes foram os ensinamentos do Mestre, transmitidos às gerações seguintes pelos seus discípulos diretos (A. Tolosa, P. W. Longo, O. Lange e C. Gama). Plasmado na Escola de Charcot e de Dejerine, discípulo de Babinski, Enjolras Vampré soube tornar a Enfermaria da $1^{*}$ Medicina de Homens na Santa Casa, onde possuia alguns leitos na enfermaria de Clínica Geral, a meca para onde se dirigiam as atenções dos estudantes que passaram pela nossa Faculdade de Medicina de 1925 a 1938, quando então uma fatalidade fêz com que Ele desse aos seus alunos uma última aula de neurologia, sofrendo um enfarte cerebral em plena classe.

São de seu Serviço várias das teses de doutoramento defendidas perante a Faculdade de Medicina que merecem figurar com ênfase na bibliografia neurológica paulista $16,18,24,25,33$.

Não é possivel deixar de se fazer menção especial ao espírito universitário de Enjolras Vampré, traduzido entre outras nesta passagem singular que foi o seu concurso para a Cátedra de Neurologia em 1935, concurso êsse que foi levado a efeito por insistência pessoal sua, Ele, que era nessa época um professor consagrado e mestre indiscutível da neurologia paulista.

Depois do desaparecimento do Mestre, a sua Escola continuou pujante, dando ramos diversos com o preenchimento, por discípulos seus, das Cátedras de Clínica Neurológica da Faculdade de Medicina da Universidade de São Paulo, da Escola Paulista de Medicina e, mais recentemente, das Faculdades de Medicina de Ribeirão Prêto e de Sorocaba. O ensino da neurologia nela se aperfeiçoou mais e mais adaptando-se ao próprio desenvolvimento da ciência, os trabalhos de pesquisa clínica se sucederam, novas subespecialidades foram criadas ou desenvolvidas. Para citar sòmente algumas, lembremos o laboratório especializado para o exame do líquido cefalorraqueano, a neurorradiologia, iniciada e cultivada com tanto carinho em São Paulo pelo pranteado Celso Pereira da Silva, a eletrencefalografia, a neuropediatria, os serviços especializados para traumatologia cranioencefálica e raquimedular.

$\mathrm{Na}$ história da neurologia paulista merece um lugar de excelso destaque a figura de Fausto Guerner. Quem o conheceu no $5 .^{\circ}$ Pavilhão do Hospital de Juqueri, certamente se lembrará de sua figura sempre irrepreensivel em seus trajes e em suas maneiras, cortês e delicado para os que dêle se acercavam, mas sempre sabendo impor aquêle respeito que se deve aos Mestres. Era êle um entusiasta da patologia cerebral, um entu- 
siasta do ensino, sempre satisfeito em se ver cercado de discípulos, sabendo lhes transmitir, com sua palavra fácil e elegante, os conhecimentos mais profundos relativos a essa patologia. Muitos se lembrarão ainda o quanto, quando internos de Juqueri, aguardavam ansiosamente pela terça-feira, o plantão semanal de Fausto Guerner, pois que nesse dia, ao lado de ensinamentos ministrados do modo o mais proficiente, ouviam-no, embevecidos, discorrer sôbre assuntos dos mais diversos setores do saber humano. Da sua capacidade de fazer discípulos, de criar núcleos de aprendizado e de trabalho cientifico, do ardor que sabia imprimir a novas pesquisas e ao emprêgo dos novos métodos de semiologia ou de terapêutica neuropsiquiátrica, muitos trabalhos científicos pioneiros se fizeram em nossa terra.

Em citando os pioneiros do estudo e do ensino da neuropsiquiatria em São Paulo, o nome de Aníbal Silveira ${ }^{34}$ desponta como um marco indelével. Foi êsse moço tímido, nem sempre compreendido nos ambientes em que labutou, conhecedor o mais profundo das funções cerebrais, discípulo das obras magistrais de Gall e de Kleist, aquêle que reuniu em tôrno de si, em gerações sucessivas, discipulos entusiastas que souberam se abeberar em tão rica fonte de conhecimentos. A sua influência se fêz sentir de modo nítido nos nossos conhecimentos a respeito da mais fina fisiopatologia cerebral, assim como da semiologia e da patologia dos órgãos corticais, em particular, as do lobo frontal.

Na década de 1930 e nas seguintes, os conhecimentos sôbre patologia cerebral, os meios de diagnóstico das doenças que afetam o sistema nervoso central, os métodos de terapêutica para o alivio dos infelizes portadores de afecções mentais ou neurológicas, sofreram transformação fundamental. A nossa geração foi, de um modo singular, presente a cada um dos novos métodos que iam surgindo e é com justificada ufania que podemos proclamar que aqui em São Paulo - na escola neurológica de Vampré e na escola neuropsiquiátrica de Juqueri — êsses métodos foram utilizados um a um, em quase primazia, à medida que iam sendo divulgados. A ventriculografia já começara a ser feita em 1930 e $1931{ }^{21,23}$, a pneumencefalografia já tomara impulso substancial em 1933, tornando-se método quase de rotina no Hospital de Juqueri, pelo entusiasmo moço de Fausto Guerner, José Fajardo e Celso Pereira da Silva ${ }^{31,32}$. A arteriografia cerebral a Egas Moniz, tìmidamente iniciada em $1928^{16}$, quando da estada do mestre português em nosso País, já em Juqueri era praticada desde $1936^{38,45}$, com primazia em todo o hemisfério sul. A ventriculografia com os óleos iodados tiveram ali, também, a sua aplicação imediata desde $1936^{40}$. A leucotomia cerebral, como método de terapêutica para certas síndromes mentais, era também em Juqueri levada a efeito em $1936^{37}$, isto é, no mesmo ano em que foi publicada pelo seu ideador, em trabalho que lhe valeu o Prêmio Nobel da Medicina. Nessa mesma seqüência, como pioneiros, aí já se iniciava a utilização da insulinoterapia de Sakel em $1936^{41}$ e a convulsoterapia de von Meduna em 193742, 43, 44, assim como em São Paulo se praticava a malarioterapia em $1927^{14}$, 15, a convulsoterapia pelo método Cerletti em $1941^{51}$, a penicilinoterapia na neurolues em $194652,53,54,55$, o tratamento efetivo da meningite tuberculosa em $1947^{58}$ e o da criptococose do sistema nervoso em 195968 .

O exame do líquido cefalorraqueano, iniciado também em Juqueri em $1923^{19}$, encontrou no espírito incentivador de Vampré, e em O. Lange os verdadeiros criadores desta nova modalidade de semiologia neurológica 22, 35, 36, 47, não só firmando o uso sistemático da punção suboccipital, e com isso tornando êsse um método de rotina, como também incentivando seus discípulos no estudo mais acurado, procurando sempre reações novas e métodos mais precisos de dosagem. Tal escola tornou possivel a São Paulo se constituir em um dos centros mais avançados do mundo neste particular. Os estudos da fisiologia cerebral com os métodos eletrônicos repercutiram em nosso meio muito cedo e a eletrencefalografia, quando ainda em seu nas- 
cedouro - por volta de 1938 - já encontrava eco no meio neurológico de Juqueri. Mesmo retardados com as vicissitudes da segunda guerra mundial já se podia em 1942-43, com o apoio o mais compreensivo do meio neuropsiquiátrico paulista, lançar as bases do seu emprêgo em São Paulo, o qual se iniciou efetivamente em 1944, isto é, sòmente precedido por Balado, na Argentina, em todo o hemisfério sul 56, 59. Hoje em dia todos os métodos semiológicos e terapêuticos da moderna neurologia, quer sejam êles clínicos ou cirúrgicos, são exercidos com plena desenvoltura nos diversos serviços neurológicos em São Paulo. A semente plantada pelos nossos ancestrais medrou e floresceu de modo a não nos deixar envergonhados perante êles ${ }^{60}$, $61,62,63,64,65,66,6 i$.

Além das "Memórias do Hospital de Juqueri", cuja publicação foi iniciada em 1924 por A. C. Pacheco e Silva e dos "Arquivos da Assistência a Psicopatas" que a sucederam em 1936, tiveram papel de realce no incentivo ao estudo da neurologia em São Paulo, a "Revista de Neurologia e Psiquiatria" e os Arquivos De Neuro-Psiquiatria. Aquela, fundada por James Ferraz Alvim e um grupo de entusiastas colaboradores em 1934, publicou material científico de grande interêsse, assim como publicou tôda a atividade científica do Departamento de Neuro-Psiquiatria da Associação Paulista de Medicina; sua vida foi curta (1934-1941) mercê dos problemas materiais criados com o advento da segunda guerra mundial. Os ARQUivos DE Neuro-Psiquiatria, pujante organização técnica e científica, comemora agora o seu vigésimo ano de atividades ininterruptas, tendo sido publicados os seus fascículos e volumes rigorosamente em datas pré-determinadas. Nascidos em reunião informal dos Profs. A. Tolosa e P. W. Longo, nos primórdios de 1943, com um grupo de assistentes das Cátedras de Neurologia da Faculdade de Medicina da USP e da Escola Paulista de Medicina, com a finalidade principal de servir de veículo aos trabalhos científicos dessas duas cátedras, assim como ao intercâmbio delas com o Exterior, tiveram os ARquivos dE NeURo-Psiquiatria, em Oswaldo Lange, um timoneiro seguro que o guiou com mão forte nesses primeiros 20 anos de sua existência.

A neurologia se tornou uma especialidade bastante complexa. Já não nos bastam a anamnese cuidadosa no sentido de identificar cada um dos sinais e dos sintomas de que é tão rica a patologia neurológica, de codificá-los no sentido cronológico com o fim de estabelecer um diagnóstico topográfico o mais acurado, de conhecer a patologia geral no sentido de nela colocar a patologia nervosa correspondente, a qual na grande maioria dos casos é dela parte, de compreender o doente e avaliá-lo no sentido psicossomático o mais profundo, para poder diferenciar bem quais são as manifestações orgânicas de um quadro mórbido, mas é preciso que tenhamos em mãos os aparelhamentos e métodos de diagnóstico os mais complexos: o laboratório de liquido cefalorraqueano com tôda a sua gama de métodos os mais finos, os meios para a pneumencefalografia em tôdas as suas variantes, para as arteriografias cerebrais, os que nos permitem o estudo elétrico do cérebro quer com a eletrencefalografia, quer com a eletrocorticografia e o registro da atividade elétrica cerebral em profundidade, e, já mais recentemente o ultrassom e os isótopos radioativos.

A neurologia deixou de ser aquela admirável especialidade cujos diagnósticos clínicos eram sỏmente precisos sob o ponto de vista da minúcia topográfica, para se tornar um dos setores mais avançados da moderna medicina, onde o diagnóstico é integral e os meios de terapêutica progridem dia a dia, competindo em bons resultados com os de qualquer outro setor da medicina.

Entretanto, tudo isso fêz com que a neurologia se tornasse uma especialidade dispendiosa e pouco acessivel à maioria dos pacientes. Para que todos êsses elementos possam funcionar harmônicamente, para que todos estejam em tempo ao alcance dos doentes neurológicos, necessário se torna 
que estejam agrupados em um único estabelecimento hospitalar. A neurologia é imperiosamente uma especialidade a ser exercida dentro de institutos especializados. Aí os doentes encontrarão, com economia de tempo indispensável ao bom êxito terapêutico e com economia real, imprescindivel para a maioria dêles em nosso meio, os meios para um diagnóstico completo e precoce, assim como para uma terapêutica eficaz, já que aplicada imediatamente. Ai os estudantes de medicina e os médicos recém-formados encontrarão o ambiente indispensável para a sua especialização em neurologia ou em qualquer dos ramos afins (neurocirurgia, laboratório clínico, neurorradiologia, eletrônica aplicada à neurocirurgia, etc.). Sòmente com Institutos de Neurologia devidamente organizados, com aparelhamento adequado e com pessoal especializado, de preferência anexos às Escolas de Medicina, poderemos daqui por diante progredir no melhor conhecimento das doenças neurológicas, que estão longe de serem raras neste País, do melhor meio de as combater, quer profilàticamente, quer curativamente, de preparar os novos especialistas que terão sôbre seus ombros a responsabilidade de levar a Neurologia paulista e brasileira além do muito que ela já caminhou. Também anexos a êsses institutos é que poderão se desenvolver centros de recuperação, quer psicológica quer física, para a legião dos infelizes que foram curados em sua moléstia, mas que desgraçadamente quedaram com seqüelas mais ou menos severas, os centros de serviço social e os de profilaxia, os de educação e os de readaptação profissional, hoje em dia cada vez mais necessários, para atender uma parcela ponderável daqueles que foram doentes neurológicos.

\title{
BIBLIOGRAFIA DA NEUROLOGIA PAULISTA (PIONEIRA)
}

\author{
EM ORDEM CRONOLÓGICA
}

1. SENG, W. - Tumor do cerebelo (5 casos operados). Bol. Soc. Med. Cir. São Paulo, 2:218-220, 1911. 2. PIRES DE CAMPOS, O. — Sôbre um caso de tumor da fossa cerebral média. Rev. Med. de São Paulo 2:94-109, 1916. 3. PIRES DE CAMPOS, O. - Fisiopatologia da linguagem. Rev. Med. de São Paulo 3:140-165, 1916, 4. VAMPRE, E. - Um caso de intoxicação por Cysticercus cellulosae. Ann. Paulistas Med. Cir., 6:97-108, 1916. 5. ALMEIDA PRADO, A. de - Tumor do pedúnculo cerebral; síndrome de Weber. Bol. Soc. Med. Cir. São Paulo 1:22-23, 1918. 6. PACHECO E SILVA, A. C. - Contribuição ao estudo da epilepsia provocada pela cisticercose cerebral. Bol. Soc. Med. Cir. São Paulo 4:223-226, 1921. 7. SENG, W. - Cirurgia cerebral. Bol. Soc. Med. Cir. São Paulo 4:119-120, 1921. 8. CAMARGO, A. C.; VAMPRE, E. - Neuralgia facial: extirpação do gânglio de Gasser. Bol. Soc. Med. Cir. São Paulo 3:363-364, 1920-1921. 9. PACHECO E SILVA, A. A. - Anatomia patológica de alguns tumores cerebrais. Bol. Soc. Med. Cir. São Paulo 4:173-175, 1921. 10. PEREIRA GOMES, J. - Valor do sinal de Foster Kennedy no diagnóstico dos tumores do lobo frontal. Bol. Soc. Med. Cir. São Paulo 4:160163, 1921. 11. ALMEIDA PRADO, A. de - Sindromes Cerebelares Mixtos. Dobici \& Cia., Rio de Janeiro, 1923. 12. PACHECO E SILVA, A. C. - Contribuição para - estudo do Treponema pallidum no córtex cerebral dos paraliticos gerais. Memórias do Hospital de Juqueri, 1:19-36, 1924. 13. VIEIRA FILHO, J. - Contribuiçāo ao estudo da paraplegia espasmódica familiar. Tese de doutoramento. Fac. Med. de São Paulo, 1925. 14. VAMPRÉ, E.; LONGO, P. W. - Malarioterapia na paralisia geral. Bol. Soc. Med. Cir. São Paulo 10:155-160, 1927. 15. PACHECO E SILVA, A. C.; PASSOS, L. - A malarioterapia na paralisia geral. Bol. Soc. Med. Cir. Sāo Paulo 11:153-155, 1927. 16. RIBEIRO, H. N. O. - Encefalografia arterial. Tese de doutoramento. Fac. Med. de São Paulo, 1928. 17. PINTO CESAR, E. - Da catatonia e suas relaçôes com os núcleos optoestriados. Tese de doutoramento. Fac. Med. de São Paulo, 1928. 18. GONÇALvES, G. - Contribuição ao estudo das sindromes da medula sacra, cone, epicone e das hipuropatias. Tese de doutora- 
mento. Fac. Med. de São Paulo, 1928. 19. PACHECO E SILvA, A. C.; CHIPIAKOFF, B. - Contribuição para o estudo do liquor em patologia mental. Memórias do Hospital de Juqueri 6:1-38, 1928-1929. 20. GAMA, C. — Alcoolização do gânglio de Gasser. Bol. Soc. Med. Cir. São Paulo 12:363-368, 1929. 21. BERNARDES DE OLIVEIRA, A. - Ventriculografia. Brasil Médico 13:1374, 1930. 22. VAMPRÉ, E.; LANGE, O. - Reaçōes do líquido cefalorraqueano: noçōes indispensáveis aos práticos. Bol. Soc. Med. Cir. São Paulo 13:213-216, 1930. 23. MONTENEGRO, J. - A propósito de dois casos de pneumencefalografias. Bol. Soc. Med. Cir. São Paulo 15: 298-309, 1931. 24. OLIVEIRA CARVALHO, P. E. - Acidentes neuro-paraliticos advindos no decurso ou pouco depois da vacinação anti-rábica. Tese de doutoramento. Fac. Med. de São Paulo, 1931. 25. WEY DE MAGALHĀE, C. - Contribuição para o estudo clínico do reflexo cutâneo plantar nas crianças. Tese de doutoramento. Fac. Med. de São Paulo, 1931. 26. MONTENEGRO, J. - Pneumencefalografias. Iev. Assoc. Paul. Med., 1:315, 1932. 27. MONTENEGRO, J.; FAJARDO, J. A. A patogenia da epilepsia à luz dos estudos pneumencefalográficos. Bol. Soc. Med. Cir. São Paulo 17:70-73, 1933. 28. VAMPRÉ, E. - Neurocirurgia em São Paulo. Rev. Assoc. Paul. Med., 2:252, 1933. 29. GAMA, C. - Estudo clínico-radiológico dos tumores da região craniofaríngea. Rev. Assoc. Paul. Med., 5:285, 1934.30. VAMPRÉ, E. - Radioterapia profunda nos traumatismos fechados do crânio e da medula. São Paulo Médico 6:161-170, 1934. 31. GUERNER, F.; FAJARDO, J.; YAHN, M.; PEREIRA DA SILVA, C. - Estudos encefalográficos em esquizofrênicos. Memórias do Hospital de Juqueri 12:195-224, 1934. 32. PTMENTEL, E. C. - Contribuição para o estudo do prognóstico da paralisia geral pela pneumencefalografia. Memórias do Hospital de Juqueri 12:61-118, 1934. 33. FONSECA BICUDO Jr., J. - O reflexo cremastérico: sua dissociação nas moléstias nervosas (sinal de Tolosa). Tese dé doutoramento. Fac. Med. São Paulo, 1934. 34. SILveIrA, A. — As funções do lobo frontal. Rev, Neurol. e Psiquiat. de São Paulo 1:196-228, 1935.35. LANGE, O. - Considerações sôbre a eosinofilia do liquido cefalorraqueano. Rev. Neurol. e Psiquiat. de São Paulo 1:421-429, 1935. 36. LANGE, O. - O líquido cefalorraqueano na cisticercose do sistema nervoso central. Rev. Neurol, e Psiquiat. de São Paulo 2:52-60, 1936. 37. MATTOS PIMENTA, A. de - Leucotomia cerebral. Arq. Assist. Psicop. de São Paulo 1:259-266, 1936. 38. BAPTISTA, V.; MATTOS PIMENTA, A. de - Tumor cerebral em menina de 7 anos (caso com arteriografia). Rev. Neurol. e Psiquiat. de São Paulo 2:388, 1936. 39. VILlAÇA, C. - Ventrículo, encéfalo e mielografias com Thorotrast. Rev. Neurol. e Psiquiat. de São Paulo 2:323-335, 1936. 40. MATTOS PIMENTA, A. de; PEREIRA DA SILVA, C. - Iodoventriculografia. Rev. Neurol. e Psiquiat. de São Paulo 3:55-56, 1937.41. YAHN, M.; ARRUDA, J. - O tratamento pelo coma insulínico em dois casos de esquizofrenia catatônica. Arq. Assist. Psicop. de São Paulo 2:99-121, 1937.42. ALMEIDA PRADO, J. N. - Contribuição para o estudo de convulsoterapia. Epilepsia experimental, Amonioconvulsiogênese. Arq. Assist. Psicop. de São Paulo 2: 475-498, 1937. 43. MARQUES DE CARVALHO, H.; SILVA, P. A. - O tratamento convulsivante da esquizofrenia: método de von Meduna. Arq. Assist. Psicop. de São Paulo 2:539-552, 1937. 44. SILVEIRA, A. - Contribuição para o tratamento convulsivante nos esquizofrênicos. Arq. Assist. Psicop. de São Paulo 2:391-450, 1937. 45. MATTOS PIMENTA, A. de; PEREIRA DA SILVA, C. - Arteriografia cerebral. Rev. Paul. Med., 11:342, 1937. 46. MAFFEI, W. E. - Tumores do sistema nervoso. Rev. de Neurol. e Psiquiat. de São Paulo 3:65-106, 1937. 47. LANGE, O. - O Liquido Cefalorraqueano em Clínica. Cia. Melhoramentos São Paulo, 1937. 48. MAFFEI, W. E. - Neoplasias meníngeas. Rev. de Neurol. e Psiquiat. de Sāo Paulo 4:1-39, 1938. 49. TOLOSA, A. - Estudo clínico dos reflexos espondileus, particularmente do reflexo espôndilo-crural. Tese de professorado. Fac. Med. da Univ. de São Paulo, 1938. 50. MAFFEI, W. E. - Tumores dos nervos periféricos. Tese de docência. Fac. Med. Univ. de São Paulo, 1940. 51. PACHECO E SILVA, A. C. O eletrochoque no tratamento das doenças mentais. Rev. de Med. Sāo Paulo 25: 15-25, 1941. 52. LONGO, P. W.; ROBORTELLA, M.; REIS, J. B. - Penicilinoterapia em seis casos de neuroluéticos já malarizados. Arq. Neuro-Psiquiat., 4:47-54, 1946. 53. LONGO, P. W.; BITTENCOURT, J. M. T. - Penicilinoterapia em um caso de 
neurite óptica luética. Arq. Neuro-Psiquiat., 4:55-59, 1946. 54. ROSSETO, O.; CAETANO DA SILVA Jr., J. A. - Penicilinoterapia na paralisia geral progressiva. Arq. Neuro-Psiquiat., 4:60-65, 1946. 55. BITTENCOURT, J. M. T.; CAETANO DA SILVA Jr., J. A.; CANELAS, H. M. - Penicilinoterapia intra-raquidia: reações imediatas e tardias. Arq. Neuro-Psiquiat., 4:68-71, 1946. 56. PUPO, P. P.; MATTOS PIMENTA, A. de - A eletrencefalografia nos tumores intracranianos. Arq. NeuroPsiquiat., 4:373-390, 1946. 57. JULIÃO, O. F. - Contribuição para o diagnóstico clinico da lepra nervosa. Tese de docência. Fac. Med. da Univ. de São Paulo, 1946. 58. MURSA, M.; REIS, J. B. dos; WOISKI, J. R. — Meningite tuberculosa tratada com estreptomicina. Arq. Neuro-Psiquiat., 5:135-146, 1947. 59. PAZZANESE, O.; PUPO, P. P. - A eletrencefalografia na epilepsia: considerações sôbre 271 casos. Arq. Neuro-Psiquiat., 6:107-150, 1948. 60. LEFEVRE, A. B. - Contribuição para a padronização do exame neurológico do recém-nascido normal. Tese de docência. Fac. Med. Univ. de São Paulo, 1950. 61. LEFEVRE, A. B. - Contribuição para o estudo da psicopatologia da afasia em crianças. Tese de doutoramento. Fac. Med. da Univ. de São Paulo, 1950. 62. LONGO, P. W.; PUPO, P. P.; PAZZANESE, O.; MATTOS PIMENTA, A. de; LEMMI, O. - A eletrocarticografia como meio de orientação na terapêutica cirúrgica da epilepsia. Arq. Neuro-Psiquiat., 10:365-373, 1952. 63. MATTOS PIMENTA, A. de; MANGABEIRA ALBERNAZ F॰, P.; BALBO, R. J. - Cisternografia nas lesões cirūrgicas da região optoquiasmática. Rev. Paul. Med., 47:468, 1955. 64. MATTOS PIMENTA, A. de - A pneumencefalografia nas lesões da fossa posterior. Apresentado à $1^{\text {a }}$ Reunião Brasileira de Neurocirurgia (Pôrto Alegre), abril de 1956. 65. REIS, J. B. dos; BEI, A. - Reação de fixação de complemento para diagnóstico da sifilis e da cisticercose no liquido cefalorraqueano pela técnica de Wadsworth-Maltaner-Maltaner. Rev. Paul. Med., 53:439-478, 1958. 66. SPINA-FRANCA, A. - Eletroforese em papel das proteínas no líquido cefalorraqueano: técnica. Arq. Neuro-Psiquiat., 16:236-242, 1958. 67. ZACLIS, J. Visibilizaçāo radiográfica de todo o sistema vascular encefálico mediante injeçāo de contraste em uma única artéria: pan-angiografia cerebral. Tese de docência. Fac. Med. da Univ. de São Paulo, 1959. 68. GIORGI, D.; REIS, J. B. dos; PUPO, P. P.; CAMARGo LIMA, J. G. - Tratamento da criptococose do sistema nervoso pelo Amphotericin B. Arq. Neuro-Psiquiat., 17:377-386, 1959.

Av. Angélica, 1920, São Paulo — Brasil. 